Parcours de recherche en SIC: littérature scientifique, méthodes et terrain

\title{
Construction d'un dispositif méthodologique et de ses outils : savoir commun et savoir scientifique, de l'induction aux hypothèses
}

Constructing a Methodological Device and Its Tools: Shared Knowledge and

Scientific Knowledge from Induction to Hypotheses

\section{Agnès d'Arripe}

\section{(2) OpenEdition}

Journals

Édition électronique

URL : http://journals.openedition.org/edc/904

DOI : $10.4000 /$ edc. 904

ISSN : 2101-0366

Éditeur

Université Lille-3

\section{Édition imprimée}

Date de publication : 1 avril 2009

Pagination : $97-108$

ISBN : 978-2-917562-01-7

ISSN : $1270-6841$

\section{Référence électronique}

Agnès d'Arripe, "Construction d'un dispositif méthodologique et de ses outils : savoir commun et savoir scientifique, de l'induction aux hypothèses », Études de communication [En ligne], 32 | 2009, mis en ligne le 01 avril 2011, consulté le 19 avril 2019. URL : http://journals.openedition.org/edc/904 ; DOI : 10.4000/edc.904

Ce document a été généré automatiquement le 19 avril 2019.

(c) Tous droits réservés 


\title{
Construction d'un dispositif méthodologique et de ses outils : savoir commun et savoir scientifique, de l'induction aux hypothèses
}

\author{
Constructing a Methodological Device and Its Tools: Shared Knowledge and \\ Scientific Knowledge from Induction to Hypotheses
}

\section{Agnès d'Arripe}

Nous désirons expliciter ici la construction du dispositif méthodologique élaboré dans la phase de récolte des matériaux nécessaires à notre travail de thèse. A travers cette recherche inscrite en sciences de la communication, nous allons nous interroger sur les différentes variables qui vont interagir pour, partant d'acteurs très différents, arriver à une nouvelle micro-culture de projet ou d'organisation. Notre terrain de recherche participe de l'Institut «handicaps, dépendance et citoyenneté » mis en place par l'Université Catholique de Lille. Cet institut est défini par ses concepteurs comme un espace de connaissances, de recherche, d'enseignements-formations et de pratiques réunissant d'une part des universitaires de toutes les disciplines scientifiques dont est dotée l'Université, et d'autre part des acteurs de terrain, du monde sanitaire et du monde médico-social et social, œuvrant dans le domaine des handicaps et de la dépendance. Des ateliers de recherche, qui constituent notre terrain d'investigation, y ont été mis en place autour de trois thématiques : vulnérabilité et fragilité ; participation sociale et autonomie ; évolution des métiers, articulation sanitaire et médicosocial. Des chercheurs de différentes branches de connaissances scientifiques ou intellectuelles, que nous nommerons ici disciplines (médecine, éthique, sociologie, philosophie, droit, ...), des acteurs de terrain (cadres de santé, médecins, ergothérapeutes, ...) et des membres d'associations liées aux thématiques de l'Institut se réunissent autour de ces trois thématiques qui font l'objet d'ateliers distincts animés par trois équipes différentes 
composées en majorité de chercheurs. L'objectif affiché est de produire ensemble un savoir, voire des travaux de recherche.

Pour fonctionner ensemble et maintenir une certaine cohérence, le groupe a besoin de partager certaines règles communicationnelles. Nous tenterons d'identifier ces règles implicites qui régissent les relations entre les participants aux ateliers de recherche. Pour cela, nous allons nous centrer sur les interactions. Ces dernières servent souvent à confirmer la relation et l'appartenance à une même culture. Comme le signale Y. Winkin ${ }^{1}$, si tout se passe normalement, on "performe" les codes de la culture à laquelle on appartient et cette co-performance a pour effet de nous rassurer quant à notre qualité de membre. La communication serait, d'un point de vue anthropologique, la «performance de la culture » ce qui s'oppose à la vision télégraphique de la communication qui était vue comme une «transmission de messages ». R. Birdwhistell, un des pères fondateurs de l'anthropologie de la communication disait qu'être membre d'une culture, c'est être prévisible. W. Goodenough définissait quant à lui la culture comme «tout ce qu'il faut savoir pour être membre ».

3 Nous nous interrogerons également sur la manière dont les acteurs métacommuniquent sur ces interactions. Nous nous intéresserons donc à leurs explications et à leurs interprétations par rapport à ce qu'ils vivent et aux échanges auxquels ils participent. Pour y parvenir nous avons choisi de travailler avec trois méthodes que nous jugeons complémentaires : l'observation participante, la méthode d'analyse en groupe et les entretiens semi-directifs. Il nous semble important de préciser d'emblée que nous nous inscrivons, ici, dans une démarche qualitative compréhensive. Plus que de mesurer, comparer ou décrire systématiquement,... nous avons, en effet, pour but de comprendre, d'identifier des comportements ou des processus.

\section{Savoir expert versus savoir profane}

4 A travers notre démarche, nous nous éloignons quelque peu de la rupture épistémologique au sens classique du terme qui voudrait que le savoir profane, appelé aussi savoir commun ou social, et le savoir expert, que nous assimilerons ici au savoir scientifique, soient séparés par des frontières étanches. B. Wynne ${ }^{2}$ définit les savoirs experts comme des connaissances standardisées, générales et abstraites qui permettent l'action à distance. Les savoirs profanes sont, eux, des savoirs faits de connaissances concrètes, locales donc fortement diversifiées et s'appliquant à une réalité dense et multidimensionnelle. Le savoir profane ou commun est donc bien loin d'un "nonsavoir ». Nous partons du postulat que l'homme contemporain produit une connaissance réflexive sur lui-même et sur ses propres expériences. Il élabore ses propres hypothèses, pistes de réflexion. Comme le signalent L. Van Campenhoudt, J-M. Chaumont et A. Franssen ${ }^{3}$, la connaissance de l'acteur sur lui-même et sur ses propres expériences, n'est toutefois pas automatiquement éclairante et valide selon les critères des sciences sociales. Plusieurs précautions devront être appliquées à l'exploitation de ce savoir profane dans le cadre d'une recherche scientifique. Une attention particulière sera apportée aux conditions de production du discours de l'acteur, ainsi qu'à ce qui, dans ce discours, est dû à la présence du chercheur.

5 Comme J.-C. Kaufmann ${ }^{4}$, nous pensons que l'objectivation doit se construire peu à peu grâce aux instruments conceptuels mis en évidence et organisés entre eux. Le rôle du 
chercheur sera également de fournir les cadres méthodologiques adéquats pour appréhender ce savoir profane. Comme le souligne également J.-C. Kaufmann, les références théoriques et conceptuelles du chercheur sont des conditions nécessaires pour continuer à apprendre de ce savoir profane quand le chercheur a délimité sa problématique avec précision et qu'il ne peut donc plus se laisser totalement guider par le discours de l'acteur sur sa pratique. Les savoirs s'élaborent dans un processus de coconstruction avec les acteurs de terrain. Nous nous placerons dans une position médiane entre le chercheur-expert, détenteur absolu $\mathrm{du}$ savoir, et la position ethnométhodologique voulant que «savoir commun" et "savoir scientifique" s'enchaînent dans une continuité parfaite. B. Dupret ${ }^{5}$ explique que, pour les chercheurs appartenant à ce courant, les individus ont des méthodes qui leur servent à prêter un sens à leur monde d'action, à s'orienter vers ce monde et à le pratiquer de manière quotidienne et routinière. L'acteur agirait comme un chercheur en sciences sociales puisqu'il analyse via l'observation, le groupe ou le contexte dans lequel il va devoir interagir.

6 L'option prise ici s'inscrit dans ce que l'on pourrait qualifier de démarche hypothéticoinductive, puisque nous ne partons pas systématiquement d'hypothèses générales à vérifier sur le terrain, mais que nous tenterons de rendre compte des expériences vécues dans toute leur complexité et leur dynamique. Le va-et-vient continuel entre les lectures et le terrain permet d'affiner et de construire progressivement une problématique solide. En travaillant dans ce mouvement, nous espérons éviter tant les écueils propres aux méthodes purement inductives que les écueils propres aux méthodes que nous qualifierons d'hypothético-déductives. À savoir : dans le premier cas, la récolte d'une masse de données dans toutes les directions conduisant finalement à une désorientation du chercheur ; dans le second, le recours à des thèses générales pouvant s'avérer réductrices, voire peu en phase avec la réalité. Pour nous, le chercheur doit arriver sur le terrain l'esprit ouvert afin de ne négliger aucune explication ou aucune direction, même si celles-ci n'étaient pas apparues lors de ses lectures préalables, mais il devra à un moment faire un choix et se concentrer sur certains aspects de la réalité.

\section{Une triangulation des méthodes}

7 Afin de pouvoir appréhender notre objet de recherche dans toute sa richesse, tout en faisant preuve de rigueur scientifique, nous avons choisi de croiser trois méthodes: l'observation participante, la méthode d'analyse en groupe et les entretiens semidirectifs. Ce choix découle des références théoriques retenues.

\section{L'observation participante}

8 Nous suivrons lors de nos recherches des auteurs comme E. Goffman, J-C. Kaufmann ou encore Y. Winkin. Comme ces auteurs, nous pensons que les normes sociales, les cultures et les identités se construisent en action et durant les interactions. Pour E. Goffman, les comportements quotidiens des hommes sont négociés par chacun dans une sorte d'improvisation permanente. Cette improvisation se soumet toutefois à des règles qui conduisent les négociations et permettent à l'ordre social de s'établir dans la vie quotidienne. 
9 Nous allons nous intéresser au «banal », à ces comportements qui nous semblent naturels et « simplement existants » et qui sont en réalité guidés par une série de règles implicites permettant de structurer la vie en société. En nous détachant quelque peu de E. Goffman, nous faisons l'hypothèse que ces règles implicites s'acquièrent lors de la socialisation au sein de différents groupes d'appartenance que nous qualifierons de " micro-culture » au sens de l'anthropologie de la communication, c'est-à-dire de lieux où les individus sont prévisibles les uns pour les autres. Nous allons considérer que l'acteur social est pluriel et qu'il va se construire une identité grâce à sa socialisation au sein de différents groupes. Ces multiples socialisations vont lui donner des indications sur la manière dont il est attendu qu'il se comporte au sein d'une situation donnée. Dans le cas qui nous occupe, trois types de socialisation nous semblent pertinents. Premièrement, l'appartenance sociale, liée au milieu social, au groupe social de référence. Deuxièmement, l'appartenance sectorielle, liée au secteur d'activité (santé, recherche, social,...). Et troisièmement, l'appartenance catégorielle, liée à la position occupée par la personne au sein de l'organisation considérée (employé, cadre, dirigeant,...). Les personnes d'une même appartenance sectorielle, catégorielle ou sociale partageraient donc aussi certains rites d'interaction et certains codes communicationnels communs, différents de ceux partagés par les autres groupes. Une certaine socialisation s'opèrerait en leur sein et des logiques communicationnelles et stratégiques propres se mettraient en place. Cette socialisation s'opérationnalise, se consolide ou se modifie lors des interactions.

10 À travers l'observation participante des ateliers de recherche et des réunions de travail préparant ces ateliers, nous espérons identifier ces éventuels rites d'interaction et codes communicationnels implicites communs aux personnes appartenant aux mêmes groupes. Nous espérons identifier également les confrontations qui pourraient exister entre des individus ne partageant pas les mêmes « codes ». En étudiant l'ordre de l'interaction et la structure de l'expérience, nous nous interrogerons sur les différentes variables qui vont peser sur la micro-culture propre aux ateliers de recherche. Quels seront ses propres rites d'interaction, son propre «langage» ? Il s'agira d'identifier la «configuration ponctuelle» que l'on retrouvera ici comme résultante de la confrontation spécifique entre ces trois groupes d'appartenance.

11 L'observation participante permet de se décentrer de son propre point de vue et de comprendre de l'intérieur les logiques et les langages des acteurs observés. Il s'agit d'une sorte d'apprentissage progressif de ces logiques et langages en étant immergé dans le quotidien des acteurs. L'observation permet de dépasser l'opinion que les acteurs ont de leur propre pratique. Comme le disent M. Grosjean et M. Lacoste : «le thème est trop exposé aux rationalisations, comme à l'oubli des détails; il est également souvent frappé d'insignifiance aux yeux des acteurs, du fait de la routine qui entoure les événements quotidiens ${ }^{6}$. Même si, comme nous le verrons plus tard, nous accordons une large place au savoir des acteurs, nous pensons qu'il existe certaines choses qui peuvent apparaître signifiantes à un observateur extérieur mais qui n'apparaissent plus comme telles à la personne socialisée au sein d'un groupe.

12 Comme l'ont fait certains sociologues de l'école de Chicago, ainsi que dans leurs prolongements actuels, Y. Winkin, nous pensons qu'il est utile, pour rendre compte de la complexité et de la richesse des pratiques, de mettre en perspective opinions et comportements. Cela nous renvoie à l'importance du va-et-vient entre théorie et terrain, car la théorie va nous permettre de voir plus et plus loin. Elle nous guide également sur les points où focaliser notre attention lors de notre « plongée » au cœur du terrain. 


\section{La méthode d'analyse en groupe}

13 Au-delà de ces codes communicationnels souvent implicites, nous nous pencherons sur la manière dont les acteurs méta-communiquent sur leurs actions et leurs comportements. Dans ce cadre, nous ferons appel aux théories de L. Boltanski et $\mathrm{L}$. Thévenot ${ }^{7}$ pour tenter de comprendre les logiques d'action mises en place et la manière dont les acteurs justifient leurs actes. Pour ces auteurs, les acteurs vont justifier leurs actions de diverses manières selon les circonstances. Les formes de généralité et de grandeur ne sont pas attachées à des collectifs mais bien à des situations. Les moments où s'exprimera la justification seront ceux de remise en cause et de critique. C'est dans ces moments que la réflexivité se déploiera et que les acteurs réfléchiront sur le sens qu'ils donnent à leurs pratiques. Nous pensons, en effet, que les individus disposent d'une part de réflexivité importante sur leurs actes et leur conduite même si nous restons persuadée qu'une part de leurs comportements sont guidés par leur socialisation au sein de différents groupes.

Nous tenterons d'approcher cette méta-communication de deux manières différentes. Tout d'abord, à travers les moments où, durant les ateliers de recherche, les acteurs disent des choses de ce qu'ils font. Ensuite, nous désirons aller plus loin en suscitant également cette méta-communication via une méthode particulière, celle de l'analyse en groupe développée par L. Van Campenhoudt, J-M. Chaumont et A. Franssen. Il s'agit de réunir différents acteurs ayant participé aux ateliers et représentatifs des différentes appartenances mises au jour, puis de partir de récits concrets concernant la manière dont ils vivent ces ateliers de recherche et dont ils ressentent la place qui est la leur en leur sein. Quelques-unes de ces expériences, proposées par les acteurs, sont choisies et analysées avec le groupe. Le but n'est pas de mettre d'accord tous les participants sur une interprétation, mais bien de les mettre d'accord sur une formulation de leurs désaccords qui permettra d'élaborer, avec le groupe, de nouvelles hypothèses et des perspectives pratiques. Cette méthode nous semble particulièrement adaptée ici, dans un contexte où l'interdisciplinaire est au centre de nos analyses. Nous sommes, en effet, face à des acteurs aux appartenances variées, amenés à travailler ensemble, autour d'un même projet.

15 Cette méthode implique un choix épistémologique fort et souligné précédemment. Nous établissons ici une continuité entre les savoirs profanes et experts, puisque les capacités réflexives des acteurs sont mobilisées. La connaissance est élaborée ici à partir des catégories interprétatives des participants qui sont ébranlées et enrichies par la confrontation avec celles des autres. Le chercheur opère également un décentrement grâce à ses connaissances théoriques et, ici, grâce à sa vision globale du phénomène. Cette méthode, et cela contribue à son intérêt, met le chercheur en danger. Il se soumet, en effet, à la validation intersubjective dans le débat avec les participants. Il évite ainsi de proposer une interprétation surplombante où personne ne se reconnaîtrait. Certaines choses pourraient toutefois ne plus être remarquées ou tues par les participants pour des raisons stratégiques. C'est pour cela que nous avons choisi de travailler à l'aide de plusieurs méthodes et de nous intéresser également au contexte dans lequel se déroulent ces interactions. 


\section{Les entretiens semi-directifs}

16 Pour approcher le contexte dans lequel ces ateliers prennent place, nous avons également réalisé des entretiens semi-directifs avec les acteurs incontournables de l'Institut Handicap, Dépendance et Citoyenneté. Par acteurs incontournables, nous entendons des personnes à l'origine du projet et/ou amenées à se charger de la réalisation concrète des ateliers de recherche. Selon nous, cette démarche peut être utile afin de mettre au jour la dimension plus stratégique des interactions. Nous pensons, en effet, que dès que des individus sont mis en présence, leurs intérêts contradictoires doivent se combiner afin d'accomplir les objectifs communs qui les ont réunis. Le concept de «jeu » permet de dépasser les oppositions en articulant les stratégies opposées des partenaires en relation et le système contenant. Grâce aux logiques culturelles, les acteurs ont des indications sur la manière d'agir dans une situation donnée. E. Goffman reprendra le concept du jeu en référence à la théorie des jeux de L. Von Neumann et $\mathrm{O}$. Morgenstern. Comme le joueur, en fonction de la situation, l'acteur social engagé dans l'interaction disposera d'un certain nombre de possibilités. Y. Winkin ${ }^{8}$ évoque l'utilisation du terme "stratégie» par E. Goffman pour désigner les aspects de l'interaction pouvant être prévus, calculés, contrôlés. Chacun pourra mettre en place la stratégie qu'il désire afin de maximiser ses gains et de minimiser ses pertes.

L'analyse des différents groupes composant l'Institut, avec cette clé, nous permettra certainement de comprendre l'origine de certains rites d'interaction mis en place. Ne pas mettre de côté cette variable stratégique nous semble essentiel si nous désirons comprendre l'émergence éventuelle d'une nouvelle micro-culture propre à ces ateliers. On peut, en effet, se demander si de tels enjeux n'empêcheront pas une véritable collaboration horizontale. La micro-culture qui va se développer ne sera-t-elle pas influencée davantage par l'une des micro-cultures présente dans le groupe ? Dans nos ateliers de recherche, l'un des savoirs pourrait prendre plus d'importance que les autres. C'est également dans cette logique que nous avons réalisé les observations participantes des réunions de préparation des ateliers et des comités de pilotage évoquées plus haut.

La méthode de l'entretien semi-directif nous semble adaptée dans le cadre de cette phase, que l'on pourrait qualifier de contextualisation, car elle permet de toucher aux représentations qu'ont les acteurs de l'action à laquelle ils participent. Ici, notre objectif n'est pas de réaliser à tout prix un grand nombre d'entretiens pour obtenir des données exploitables quantitativement, mais bien de réaliser les entretiens nous-mêmes et d'insérer aussi dans notre analyse le contexte d'énonciation et une vue plus réflexive sur le rapport enquêteur/enquêté. Après un bref exposé introductif portant sur les objectifs de notre entretien, nous avons choisi de nous baser sur une liste de thèmes à aborder en formulant ceux-ci comme des questions ouvertes. Dans cette phase de notre recherche, nous souhaitons, en effet, laisser un maximum de liberté à la personne interviewée. Pour qu'elle exprime sa propre réalité avec son propre langage, ses propres concepts et ses propres catégories mentales, nous avons limité nos interventions et nous avons tenté de ne pas imposer nos propres cadres de références et nos éventuelles hypothèses. Ainsi, la grille de questions ou de thèmes mise en place a été élaborée dans le souci constant de rester générale et vague, afin d'introduire le moins possible de biais. Notons également que notre grille n'a été utilisée qu'à titre indicatif et que nous n'y sommes pas restée attachée systématiquement. Les thèmes sont parfois survenus dans le désordre, 
l'essentiel restant que tous les thèmes soient abordés par notre interlocuteur. Etant donné le niveau d'éducation et de responsabilité assez élevé des personnes interviewées, le fait de nous munir d'un tel guide nous semblait approprié. Vu la différence d'âge et notre arrivée récente sur ce terrain, la présence de ce guide nous positionnait de suite dans un rapport d'enquêteur-enquêté et légitimait notre place de chercheuse. La présence de ce guide nous permettait également de recadrer la discussion si elle s'orientait vers des thématiques trop éloignées de notre objet de recherche.

\section{Méthodologie et société de réseaux}

19 Le choix de travailler avec trois méthodes complémentaires nous semble pertinent tant vis-à-vis de notre terrain de recherche que des théories des auteurs que nous mobilisons. Il nous semble essentiel d'articuler ces différentes méthodologies afin d'aborder la situation dans toute sa complexité. Le fait même d'utiliser cette variété d'approche nous renvoie d'ailleurs à notre cadre théorique et à cette logique du réseau propre à notre société contemporaine. Tout comme L. Van Campenhoudt et al., nous pensons que «les promontoires érigés par la discipline sociologique pour observer le social sont eux-mêmes constitutifs du paysage qu'ils observent $"$.

Le simple fait que les ateliers de recherche existent et que leurs concepteurs s'interrogent sur l'interdisciplinarité nous renvoie aux cadres théoriques choisis. Si, dans la société industrielle, les sphères d'activité étaient fortement différenciées et que les fonctions étaient spécialisées, à l'ère du travail en réseau, nous sommes plus dans une logique transversale, impliquant une dédifférenciation inter-champs et inter-institutions. C'est du moins le constat que font les auteurs de la méthode d'analyse en groupe pour la sphère de l'intervention sociale, pour les professionnels de l'action publique. Nous faisons l'hypothèse que cette logique transversale peut s'étendre au-delà de cette sphère, à l'organisation en général. Comme le signalent les auteurs, cette hybridation permet de lutter contre les effets pervers des cloisonnements institutionnels et professionnels, mais elle est aussi une source de confusion des rôles et des fonctions. A l'heure actuelle, l'individu n'est plus figé dans « une » position ou dans « un » système d'ordres. Il invente son expérience plus activement. Pour F. Dubet ${ }^{10}$, dans ces circonstances, l'individu est amené à prendre distance et à produire une activité significative afin de construire son identité personnelle.

21 À une approche cloisonnée, nous opposons également, à travers notre projet de thèse, une approche rassemblant des théories différentes autour d'un même objet. Comme c'est le cas au sein du monde en réseau décrit par L. Boltanski et E. Chiapello ${ }^{11}$, c'est notre projet qui est le prétexte de la connexion et qui va activer ces liens. C'est la mobilisation de ces différents auteurs et l'articulation de leurs théories dans un cadre homogène et synthétique qui nous permettra d'interpréter les données recueillies grâce aux méthodologies mises en place.

Pour nous, les sciences de la communication sont caractéristiques, depuis leur origine, de ce travail en réseau. Elles mobilisent, en effet, en fonction des projets ou des problèmes à traiter, des auteurs venus de disciplines variées et les articulent pour former un cadre théorique cohérent et pertinent. En préservant leur spécificité qui se situe dans le regard communicationnel posé sur la problématique, les sciences de la communication se distinguent par leur capacité à mobiliser des ressources issues de disciplines variées. La 
méthodologie utilisée par les chercheurs en SIC se caractérise également, selon nous, par cette interdisciplinarité.

\section{BIBLIOGRAPHIE}

Boltanski, L., Chiapello, E., (1999), Le nouvel esprit du capitalisme, Paris, Gallimard, 843p.

Boltanski, L., Thevenot, L., (1991), De la justification. Les économies de la grandeur, Paris, Gallimard, 483p.

Dubet, F., (1987), La galère : jeunes en survie, Paris, Fayard, 493p.

Dupret, B., (2006), Le jugement en action : Ethnométhodologie du droit, de la morale et de la justice en Egypte, Genève, Droz, 491p.

Grosjean, M., Lacoste, M., (1999), Communication et intelligence collective. Le travail à l'hôpital, Paris, PUF, 255p.

Kaufmann, J-C., (1996), L'entretien compréhensif, Paris, Nathan, 126p.

Van Campenhoudt, L., Chaumont, J-M., Franssen, A., (2005), La méthode d'analyse en groupe : Applications aux phénomènes sociaux, Paris, Dunod, 215p.

Winkin, Y., (éd.) (2000 [1981]), La nouvelle communication, Paris, Seuil, 373p.

Winkin, Y., (2001 [1996]), L'anthropologie de la communication : de la théorie au terrain, Paris, Seuil, 332p.

Wynne, B., (1999), Une approche réflexive du partage entre savoir expert et savoir profane, in Les Cahiers de la sécurité intérieure, n³8, Paris, pp. 219-236.

\section{NOTES}

1. Winkin Y. (2001 [1996]), L'anthropologie de la communication : de la théorie au terrain, Paris, Seuil, 332p.

2. Wynne B. (1999), Une approche réflexive du partage entre savoir expert et savoir profane, in Les Cahiers de la sécurité intérieure, $n^{\circ} 38$, Paris, pp. 219-236.

3. Van Campenhoudt L., Chaumont J-M., Franssen A. (2005), La méthode d'analyse en groupe : Applications aux phénomènes sociaux, Paris, Dunod, 215p.

4. Kaufmann J-C. (1996), L'entretien compréhensif, Paris, Nathan, 126p.

5. Dupret B. (2006), Le jugement en action : Ethnométhodologie du droit, de la morale et de la justice en Egypte, Genève, Droz, 491p.

6. Grosjean M., Lacoste M. (1999), Communication et intelligence collective. Le travail à l'hôpital, Paris, PUF, p.47.

7. Boltanski L., Thevenot L. (1991), De la justification. Les économies de la grandeur, Paris, Gallimard, 483p.

8. Winkin Y. (dir.), (2000 [1981]), La nouvelle communication, Paris, Seuil, 373p.

9. Van Campenhoudt L. et al., op. cit, p.20. 
10. Dubet F. (1987), La galère : jeunes en survie, Paris, Fayard, 493p.

11. Boltanski L., Chiapello E. (1999), Le nouvel esprit du capitalisme, Paris, Gallimard, 843p.

\section{RÉSUMÉS}

Nous désirons expliciter la construction du dispositif méthodologique mis en place dans la phase de récolte des matériaux nécessaires à notre travail de thèse portant sur les rites d'interaction et les logiques d'action mises en place au sein de groupes interdisciplinaires. Nous commencerons par une réflexion sur le choix d'une démarche tendant vers l'induction. Nous expliquerons notre choix de travailler dans un mouvement de continuel va-et-vient entre le terrain et les approches théoriques pouvant éclairer notre problématique. Dans un second temps, nous discuterons de notre position face à la rupture épistémologique au sens classique du terme qui voudrait que savoir commun et savoir scientifique soient séparés par des frontières étanches. Nous défendrons l'idée que l'acteur produit une connaissance réflexive sur lui-même et sur ses propres expériences. Approcher ce type de savoir nécessite de solides outils méthodologiques. Nous expliquerons pourquoi nous avons choisi de croiser trois méthodes : l'observation participante, la méthode d'analyse en groupe et les entretiens semi-directifs, en justifiant ce choix en fonction de notre objet de recherche et des savoirs que ces différentes méthodes permettent d'appréhender.

We would like to clarify the construction of our methodological device which has been set up in the phase of collection of materials necessary for our thesis with regards to the interaction rituals and the logics of action within interdisciplinary groups. We will start with our choice of a thought process tending towards induction. We will work through a circular movement between field and theories that clarifies our set of questions. In a second part, we will discuss our position about the epistemological breach which states that common knowledge and scientific knowledge are separated by rigid boundaries. We will defend the idea that one produces a reflexive knowledge on himself/herself and with his/her own experiences. To approach this knowledge, we need solid methodological tools. We will explain why we chose to cross three methods: participating observation, "method of analysis in group" and semi-directive interviews. We will justify this choice according to our object of research and to the knowledge that these various methods make possible to apprehend.

\section{INDEX}

Mots-clés : dispositif méthodologique, rites d'interaction, logiques d'action

Keywords : methodological device, interaction rituals, logics of action

\section{AUTEUR}

\section{AGNÈS D'ARRIPE}

Agnès d'Arripe est enseignante-chercheure au sein de l'équipe C-SIS (ISTC- UCLille). Elle réalise actuellement une thèse de doctorat à l'Université Catholique de Louvain sous la direction de P. de 
Saint Georges et de C. Routier. Son travail porte sur l'élaboration interactive de concepts et d'outils communs, dans le cadre d'un projet interdisciplinaire. Elle s'intéresse également aux situations de communication interculturelle; aux logiques multiples de l'acteur social et à l'émergence de cultures communes. 